\title{
HOSPITAL PHARMACY PROFESSION IN NEPAL THROUGH THE EYE OF A PHARMACIST \\ R Sharma Poudel, A Prajapati
}

Hospital pharmacy, Chitwan Medical College, Chitwan, Nepal.

*Correspondence to: Ramesh Sharma Poudel, Hospital pharmacy, Chitwan Medical College, Chitwan, Nepal.

Pharmacy profession is one of the most renowned and recognized professions in the world. Hospital pharmacy is a branch of pharmacy and is well practiced in theWestern society, but in the context of Nepal, the situation is quite different. Hospital pharmacy of Nepal is usually overcrowded with patients due to limited health facilities, inadequate human resources, infrastructure and technology. Most of the hospitals further lack Drug and Therapeutic Committee (DTC), Drug information centre (DIC) and Pharmacovigilance centre, and in practice, the established DTC, DIC and Pharmacovigilance centre are functioning at sub-optimal level. The hospital pharmacy seems to be a profession of pride for many pharmacists with lots of expectation. However, the reality is that many of the pharmacist fail to meet their own expectation mainly due to lack of self competency, and appreciation by patients and other healthcare professionals. Moreover, a pharmacist may frequently encounter with weak regulation and implementation of policies, inadequate human resources, hectic dispensing focused schedule, more money-oriented organizational policy and poor images left behind by the seniors, all of which contribute to slow growth of the hospital pharmacy practice in Nepal. Also, gap has been observed between pharmacy education and current need in hospital pharmacy practice.

The understanding of patients towards pharmacy profession is poor. They often give less priority to appropriate use of medicines and medical devices. They do not seem to worry much about the side effects, interactions, contraindications, precautions to be taken and action needed to be taken in cases of missed dose. Patients are usually in rush to leave the pharmacy and frequently raise their voice to the pharmacist to draw circles and write time of administration on the primary packages of the medication (usually a permanent marker). Patients with chronic disease such as hypertension, diabetes, chronic obstructive pulmonary disease often refuse to attend the medication counseling centre despite the fact that we have found patients using their medication and medical devices incorrectly for a long time. Patients have been found to use their inhaler devices through nose and without breaking dry powder inhalers capsule, taking pessaries even through mouth and frequently visit emergency room due to exacerbation of diseases.

Collaborative efforts between health authorities and educational institutions, integration of innovative approaches in hospital pharmacy practice, enriched patient awareness on appropriate use of medicines and medical devices together with patient focused services could overcome these barriers and achieve the transition towards good hospital pharmacy practice and pharmaceutical care. Urgent academic exercise aimed at attaining revamping of curriculum, keeping C 2016, JCMC. All Rights Reserved 
Poudel et al, Journal of Chitwan Medical College 2016; 6(16)

in pace with current and emerging trends in the field REFERENCES

of hospital pharmacy practice and pharmaceutical care is needed. Further, hospital pharmacy practice should be evidence based, initiation of continue pharmacy education and innovative research on pharmacy practice need to be encouraged. Such approach may upgrade hospital pharmacy profession in Nepal in near future.

Competing interests: The authors declare that they have no competing interests.

1. Katoue MG, Awad AL, Schwinghammer TL, Kombian SB. Pharmaceutical care in $\mathrm{Ku}-$ wait: hospital pharmacists' perspectives. Int J Clin Pharm 2014;36(6):1170-8.

2. Jishnu V, Gilhotra RM, Mishra DN. Pharmacy education in India: Strategies for a better future. J Young Pharmacists 2011;3(4):334-42.

3. Katoue MG, Awad AI, Schwinghammer TL, Kombian SB. Pharmaceutical care education in

\section{Correspondence Author:}

Ramesh Sharma Poudel

Hospital Pharmacist and Member Secretary of Drug and Therapeutic Committee

Chitwan Medical College, Chitwan, Nepal Kuwait: pharmacy students' perspectives. Pharmacy Practice (Granada) 2014;12(3):411.

4. National Good Pharmacy Guidelines. Nepal Pharmacy Council. November 2005.

5. Toklu HZ. Promoting evidence-based practice in pharmacies. Integrated Pharmacy Research and Practice 2015;4:127-31.

Email: pharmacistsharma23@gamil.com 